\title{
Investigating Causal Relations Between Circulating Metabolites and Alzheimer's Diseases: A Mendelian Randomization Study
}

\section{Shu-Yi Huang}

Fudan University Huashan Hospital Department of Neurology

\section{Yu-Xiang Yang}

Fudan University Huashan Hospital Department of Neurology

\section{Ya-Ru Zhang}

Fudan University Huashan Hospital Department of Neurology

\section{Kevin Kuo}

Fudan University Huashan Hospital Department of Neurology

\section{Hong-Qi Li}

Fudan University Huashan Hospital Department of Neurology

\section{Xue-Ning Shen}

Fudan University Huashan Hospital Department of Neurology

\section{Shi-Dong Chen}

Fudan University Huashan Hospital Department of Neurology

\section{Ke-Liang Chen}

Fudan University Huashan Hospital Department of Neurology

\section{Qiang Dong}

Fudan University Huashan Hospital Department of Neurology

\section{Lan Tan}

Qingdao Municipal Hospital Group Neurology Department

Jin-Tai Yu ( $\nabla$ jintai_yu@fudan.edu.cn )

Huashan Hospital Fudan University https://orcid.org/0000-0002-7686-0547

\section{Research article}

Keywords: Metabolite, Mendelian randomization, Alzheimer's disease

Posted Date: July 19th, 2021

DOI: https://doi.org/10.21203/rs.3.rs-467119/v2 
License: (c) (i) This work is licensed under a Creative Commons Attribution 4.0 International License. Read Full License

Version of Record: A version of this preprint was published at Journal of Alzheimer's Disease on March 11th, 2022. See the published version at https://doi.org/10.3233/JAD-220050. 


\section{Abstract}

\section{Background}

Metabolomics is a promising approach that can be used to understand pathophysiological pathways of Alzheimer disease (AD). However, the relationships between metabolism and AD are poorly understood. The aim of this study is to investigate the causal association between circulating metabolites and risk of $A D$ by combining metabolomics with genomics through two-sample Mendelian randomization (MR) approach.

\section{Methods}

Genetic associations with 123 circulating metabolic traits were utilized as exposures. A large summary statistics data from International Genomics of Alzheimer's Project was used in primary analysis, including 21,982 AD cases and 41,944 controls. Validation was further performed using family history of $A D$ data from UK Biobank (27,696 cases of maternal $A D, 14,338$ cases of paternal $A D$ and 272,244 controls). We utilized the inverse-variance weighted method as primary analysis and four additional MR methods (MR-Egger, weighted median, weighted mode, and MR pleiotropy residual sum and outlier) for sensitivity analyses.

\section{Results}

We found one-fold increased risk of developing $A D$ per standard deviation increase in the levels of circulating $A p o B$ (odd ratio $(O R)=3.18 ; 95 \%$ confidence interval $(\mathrm{Cl}): 1.52-6.66, \mathrm{P}=0.0022$ ) and glycoprotein acetyls ( $\mathrm{OR}=1.21 ; 95 \% \mathrm{Cl}$ : $1.05-1.39, \mathrm{P}=0.0093)$, serum total cholesterol $(\mathrm{OR}=2.73 ; 95 \% \mathrm{Cl}$ : 1.41-5.30, $P=0.0030)$, and low-density lipoprotein $(L D L)$ cholesterol (OR=2.34; 95\% Cl: 1.53-3.57, $\mathrm{P}=0.0001)$. Whereas glutamine $(\mathrm{OR}=0.81 ; 95 \% \mathrm{Cl}: 0.71-0.92, \mathrm{P}=0.0011)$ were significantly associated with lower risk of $A D$. We also detected causal effects of several different composition of LDL fractions on increased $A D$ risk, which has been verified in validation. However, we found no association between circulating high-density lipoprotein cholesterol and AD.

\section{Conclusions}

Our findings provided robust evidence supporting causal effects of circulating glycoprotein acetyls, ApoB, LDL cholesterol, and serum total cholesterol on higher risk of AD, whereas glutamine showed the protective effect. Further research is required to decipher the biological pathways underpinning associations.

\section{Background}

Alzheimer's disease (AD) is the leading cause of dementia, affecting dramatically increased number of aging populations and placing an enormous burden on patients, families, and health-care systems [1]. The hallmarks of $A D$ include amyloid plaques, tau neurofibrillary tangles, neurodegeneration, and 
synaptic loss [1, 2]. Unfortunately, there is currently no effective prevention and treatment for AD [1]. Metabolomics is the newest systematic biology approach which measures the biochemical products of cell processes downstream of genomic, transcriptomic, proteomic systems, and influences from the environment. It offers great potential for the diagnosis and prognosis of neurodegenerative diseases by capturing snapshots of the complex and multifactorial biochemical pathways that may be altered in $A D$ [3]. Recent studies have showed that metabolomics can be used to measure alterations in biochemical pathways related to $A D[4-8]$. However, one of the key challenges in these metabolomics studies is the inability to ensure whether the relationships between circulating metabolites and $A D$ are causal. It is of great importance to understand the causality between metabolites and $A D$, as well as the potential pathophysiological pathways of $A D$, to inspire drug discovery and to detect biomarkers that aid in early detection of high-risk individuals to initiate prevention, monitoring, and treatment.

Mendelian randomization (MR) is an analytic approach that uses genetic variants as instrumental variables (IVs) to assess causal inferences between an exposure and the outcome of interest [9]. The MR approach is largely independent of unmeasured confounding biases and reverse causality inherent in observational studies, given that allocation of genotypes from parents to offspring is random and genetic variation is unlikely to be affected by environmental factors $[9,10]$. This is particularly relevant in a metabolomic study where the inter-individual variability of circulating metabolites can be affected by a wide range of potential confounders such as age, sex, physical diseases, medication, exercise, weight, and time of sampling. In addition, two-sample MR analysis is an extension in which the effects of the genetic instrument on the exposure and on the outcome can be obtained from publicly available genomewide association studies (GWAS) summary data [11]. The two-sample MR approach enables us to link circulating metabolites with risk of AD using GWAS estimates on both metabolic phenotypes and AD.

The aim of this study was to investigate the causal relationship between circulating metabolites and risk of $A D$ by applying a two-sample MR approach and combining genome-wide and metabolome-wide datasets generated from large scale cohorts.

\section{Methods}

\section{Two-sample MR design}

We inferred causal relationships between circulating metabolites and AD risk using two-sample MR, in which the selections of IVs are based on GWAS summary statistics generated from different, nonoverlapping samples. To obtain unbiased estimates of the causal effects, MR analysis rests on three assumptions [10]: (i) the genetic variants are associated with the exposure; (ii) the genetic variants are independent of confounders of the risk factor-outcome association; and (iii) the genetic variants influence the outcome only through the exposure.

\section{Genetically determined metabolites}


We obtained the summary statistics from a GWAS for 123 circulating metabolites derived from 13 cohorts $[12,13]$. Briefly, Kettunen et al. conducted a comprehensive GWAS estimated with quantitative human serum/plasma metabolites as phenotypes [12]. Metabolomics data were acquired based on human fasting blood samples, otherwise the fasting time effect were adjusted in original study. The 123 metabolites represent a broad molecular signature of systemic metabolism and were assigned to 12 classes (carboxylic acids and derivatives, fatty acyls, glycerolipids, glycerophospholipids, hydroxy acids and derivatives, keto acids and derivatives, lipoprotein, organooxygen compounds, protein, ratio, sphingolipids, steroids and steroid derivatives) based on human metabolome database classification and expert opinion $[13,14]$. A total of nearly 25,000 individuals were meta-analyzed. Written informed consent was obtained from all participants, and the study was approved by ethical committees.

\section{Selection of instrumental variables}

For each of the 123 metabolites, single nucleotide polymorphisms (SNPs) associated at genome-wide significance $P$-value $\left(P<5 \times 10^{-8}\right)$ with a minor allele frequency greater than 0.01 were considered as potential instruments. Independent SNPs were selected at a threshold of linkage disequilibrium $r^{2}>0.05$ and a distance of $1000 \mathrm{~kb}$. For palindromic SNPs, we aligned strands using allele frequency and discarded palindromic SNP(s) that had minor allele frequency above 0.42. Then exposure-outcome datasets were harmonized. We have considered the palindromic SNPs and checked original datasets to avoid reverse effects.

The proportion of variance explained by IVs were computed. And F-statistic of each metabolite was calculated to judge the strength of IVs. The bias from weak instruments depends on the strength of the instrument through the F-statistic, which is related to the proportion of variance in the phenotype

explained by IVs $\left(R^{2}\right)$, sample size $(n)$ and number of instruments $(k)$ by the formula $F=\left(\frac{n-k-1}{k}\right)\left(\frac{R^{2}}{1-R^{2}}\right)$ Typically, a strong instrument was defined as an F-statistic $>10$. Additionally, power calculations were conducted using the $\mathrm{R}$ code provided by Burgess $\mathrm{S}$ with a two-sided type-I error rate $\mathrm{a}=0.05$ [16]. Details of the genetic instruments were presented in Additional file 1: Table S1.

\section{IGAP AD dataset}

In primary analysis, genetic variants associated with late-onset AD were obtained from a meta-analysis GWAS performed by International Genomics of Alzheimer's Project (IGAP) [17]. There is no sample overlap of IGAP with cohorts of circulating metabolites. IGAP is a large two-stage study based upon GWAS on individuals of European ancestry. Data from stage 1 was used in the present study, including 63,926 individuals (21,982 AD cases and 41,944 cognitively normal controls) of European descent from four consortia: Alzheimer Disease Genetics Consortium (ADGC), Cohorts for Heart and Aging Research in Genomic Epidemiology Consortium (CHARGE), The European Alzheimer's Disease Initiative (EADI), and the Genetic and Environmental Risk in AD/Defining Genetic, Polygenic and Environmental Risk for Alzheimer's Disease Consortium (GERAD/PERADES) [17]. All AD diagnoses were autopsy-confirmed or 
satisfied the NINCDS-ADRDA criteria or DSM-IV guidelines [17]. The average age at onset of AD ranged from 74.4 to 81.9 years, and the average age at examination for $83 \%$ controls is $\geq 76$ years.

\section{UK Biobank proxy-AD dataset}

We additionally set out to validate our results using a proxy-AD dataset, based on individuals in the UK Biobank (http://www.ukbiobank.ac.uk) [18, 19]. The high heritability of AD implies that individuals with family history of $A D$ are likely to have a higher genetic $A D$ risk load. Thus, individuals with one or two parents with $A D$ were defined as proxy cases, that is, having family history of $A D$ [20]. In this dataset, the proxy-AD case-control status was ascertained via self-report. Over 500,000 community-dwelling individuals aged between 37 and 73 years were recruited in the United Kingdom between 2006 and 2010 [18]. An array of 314,278 participants with available $A D$ information on at least one parent were metaanalyzed in this analysis, including 27,696 cases of maternal $A D, 14,338$ cases of paternal $A D$, and 272,244 controls [19]. All data sources used in this MR study received approval from an ethics standards committee on human experimentation and obtained informed consent from all participants.

\section{Statistical analysis for Mendelian randomization}

We used inverse-variance weighted (IVW) method as the primary analysis to determine the causal relationships between genetically determined circulating metabolites and AD. The IVW method will return an unbiased estimate in the absence of horizontal pleiotropy or when horizontal pleiotropy is balanced [21]. Results are presented as odds ratio (OR) per standard deviation (SD) increase in genetically determined metabolites on $A D$ for the outcome was dichotomous.

We conducted sensitivity analysis using weighted median [22], weighted mode-based estimate (MBE) [23], MR Egger regression [24], and Mendelian randomization pleiotropy residual sum and outlier (MRPRESSO) [25], and displayed in scatter plots. These methods hold different assumptions at the costs of reduced statistical power. The weighted median allows for $50 \%$ of the IVs to be invalid or present pleiotropy [22]. The weighted MBE method used the mode of the IVW empirical density function as the weighted MBEs and obtained a causal effect estimate robust to horizontal pleiotropy [23]. MR-Egger regression allows $>50 \%$ of the variants to be invalid [24]. MR-Egger is based on the "NO Measurement Error" (NOME) assumption (no measurement error in the SNP exposure effects), which is evaluated by the regression dilution $\mathrm{I}_{\mathrm{GX}}$ statistic (i.e., less than 0.9 indicates a violation of NOME) [26]. Thus, an $\mathrm{I}_{\mathrm{GX}}$ statistic was calculated to test the presence of bias with MR-Egger.

Furthermore, we conducted MR Egger intercept and MR-PRESSO global test to detect the presence of horizontal pleiotropy or heterogeneity. In the case of horizontal pleiotropy, MR-PRESSO outlier test compares the observed and expected distributions of the tested variants to identify outlier variants. If significant outliers $(P<0.05)$ are detected, they were removed from the analysis to return an unbiased causal estimate [25]. Heterogeneity in the IVW estimates was tested using Cochran's Q test, and displayed in forest plots. Moreover, visual inspection of funnel plot and leave-one-out plot were also used to assess the MR "no horizontal pleiotropy" assumption (see Additional file 2: Fig S1-S5). 
To correct for multiple comparisons, we applied false discovery rates (FDR) correction in IVW. An FDR corrected P-value $<0.05$ was considered significant, and an unadjusted P-value $<0.05$ was considered as the evidence of a suggestive association. Those metabolites that showed suggestive and significant evidence of association ( $\left.P_{I V W}<0.05\right)$ with late-onset $A D$ were further assessed in validation using UK Biobank GWAS. Results with consistent direction of point estimates across sensitivity analyses, validation, IVW, and estimates after correction of potential pleiotropy were considered as robust causal associations.

Analyses were conducted using $\mathrm{R}$ version 3.6.3, with the MR analysis performed using the “TwoSampleMR" package version 0.5.2 [27, 28].

\section{Pathway enrichment analysis}

Metabolic enrichment analysis was conducted using the web-based MetaboAnalyst 5.0 (https://www.metaboanalyst.ca/). We analyzed the metabolite sets from Kyoto Encyclopedia of Genes and Genomes (KEGG) Database in our study [29]. All metabolites that were associated with AD risk with a $P$-value $<0.05$ were used to identify metabolic pathway.

\section{Results}

A flow diagram depicting the process of the MR analyses is shown in Fig 1. The IVW identified 32 circulating metabolites that were associated with $A D$ risk, including 27 significant (FDR $P_{\text {IVW }}<0.05$ ) traits and five suggestive $\left(P_{I V W}<0.05\right)$ causal traits (Fig 2). Of the 32 metabolites, the IVs were composed of 652 SNPs and could explain 3-20\% of the variance of the corresponding metabolites (see Additional file 1: Table S1). And the F-statistics were all highly above the threshold of weak instruments of F-statistic $<10$. Results of sensitivity analyses were showed in Additional file 1: Table S2. The negative MR results were presented in Additional file 1: Table S3. The list of the SNPs used as IVs for each circulating metabolite was presented in Additional file 1: Table S4.

\section{Effects of Genetically Determined Metabolites on AD}

Those associated metabolites include two proteins, two carboxylic acids and derivatives, 26 lipoproteins, and two steroids and steroid derivatives (Fig 2). We observed a 1-fold increased risk of developing AD per $\mathrm{SD}$ increase in the level of circulating $\mathrm{ApoB}\left(\mathrm{OR}=3.18 ; 95 \%\right.$ confidence interval $(\mathrm{Cl}): 1.52-6.66, \mathrm{P}_{\mathrm{IVW}}=$ $0.0022)$ and glycoprotein acetyls $\left(\mathrm{Gp} ; \mathrm{OR}=1.21 ; 95 \% \mathrm{Cl}: 1.05-1.39, \mathrm{P}_{\mathrm{IVW}}=0.0093\right)$, serum total cholesterol (serum-C; $\left.\mathrm{OR}=2.73 ; 95 \% \mathrm{Cl}: 1.41-5.30, \mathrm{P}_{\mathrm{IVW}}=0.0030\right)$ and esterified cholesterol $(\mathrm{OR}=2.42$; $\left.95 \% \mathrm{Cl}: 1.07-5.48, \mathrm{P}_{\mathrm{IVW}}=0.0345\right)$. Citrate $\left(\mathrm{OR}=0.83 ; 95 \% \mathrm{Cl}: 0.73-0.95, \mathrm{P}_{\mathrm{IVW}}=0.0055\right)$ and glutamine (OR $\left.=0.81 ; 95 \% \mathrm{Cl}: 0.71-0.92, \mathrm{P}_{\mathrm{IVW}}=0.0011\right)$ were significantly associated with lower risk of AD. In case of lipoproteins, five intermediate-density lipoprotein (IDL) subfractions (i.e., IDL-C, IDL-FC, IDL-L, IDL-P, and IDL-PL) and 15 low-density lipoprotein (LDL) subfractions (i.e., LDL-C, L-LDL-C, L-LDL-CE, L-LDL-FC, LLDL-L, L-LDL-P, L-LDL-PL, M-LDL-C, M-LDL-CE, M-LDL-L, M-LDL-P, M-LDL-PL, S-LDL-C, S-LDL-L, and S-LDL- 
P) were significantly associated with higher risk of $A D$. Four high-density lipoprotein (HDL) subfractions (i.e., XL-HDL-C, XL-HDL-CE, XL-HDL-FC, XL-HDL-P) were associated with lower risk of AD, while S-HDL-P showed association with higher risk of $A D$ at a suggestive level. Besides, XS-VLDL-PL was significantly associated with higher risk of $A D$ using IVW method $\left(O R=1.49 ; 95 \% \mathrm{Cl}: 1.11-2.02, \mathrm{P}_{\mathrm{IVW}}=0.0087\right)$.

\section{Pleiotropy and heterogeneity analysis}

The Q-test detected no evidence of heterogeneity in the results of Gp, citrate, glutamine, S-HDL-P, XL-HDLC, XL-HDL-CE, and XL-HDL-FC (Table 1). MR Egger intercept test showed no evidence of pleiotropy in these IVW-identified metabolites (Table 1). MR-PRESSO global test also showed no pleiotropy in the results of $\mathrm{Gp}$, citrate, glutamine, XL-HDL-C, XL-HDL-CE and serum-C. Others were analyzed by MRPRESSO after removing the outlying SNPs identified by MR-PRESSO outlier test. The corrected results were significant except for XS-VLDL-PL $\left(P_{\text {cMR-PRESSO }}=0.396\right)$ and IDL-FC $\left(P_{\text {CMR-PRESSO }}=0.074\right)$. The corrected point estimates of MR-PRESSO were all consistent with IVW except for XL-HDL-FC (OR ${ }_{\mathrm{CMR}}$ PRESSO $=1.64$ vs. $\mathrm{OR}_{\mathrm{IVW}}=0.90$ ). Thus, effects of XS-VLDL-PL, IDL-FC, and XL-HDL-FC were considered unreliable.

\section{Sensitivity analysis}

The results for the sensitivity analyses of 32 IVW-identified metabolites are showed in Fig 4 and Additional file 1: Table S2. The ${ }^{2}{ }_{G X}$ statistics of each MR-Egger result are all above 0.9. ApoB, Gp, glutamine, and serum- $C$ present robust associations that all additional MR methods showed consistent direction of point estimates with IVW, though not all MR methods yielded significant results. However, as for citrate, the sensitivity results showed opposite effect estimates $\left(\mathrm{OR}_{\text {weighted-median }}=1.06, \mathrm{OR}_{\text {weighted- }}\right.$ mode $=1.05)$ to primary result $\left(\mathrm{OR}_{\mathrm{IVW}}=0.83\right)$. In case of lipoproteins, an array of $16 \mathrm{IVW}$-identified significant metabolites (i.e., IDL-C, IDL-L, IDL-P, LDL-C, L-LDL-C, L-LDL-CE, L-LDL-FC, L-LDL-L, L-LDL-P, LLDL-PL, M-LDL-CE, M-LDL-L, M-LDL-P, M-LDL-PL, S-LDL-C, and S-LDL-P) also showed concordant results across sensitivity methods and IVW. Sensitivity analysis indicates non-robust effects of S-LDL-L $\left(\mathrm{OR}_{\text {weighted-median }}=0.80, \mathrm{OR}_{\text {weighted-mode }}=0.80 \mathrm{vs} O \mathrm{R}_{\mathrm{IVW}}=2.69\right), \mathrm{XL}-\mathrm{HDL}-\mathrm{CE}\left(\mathrm{OR}_{\text {weighted-median }}=1.20\right.$, $\mathrm{OR}_{\text {weighted-mode }}=1.23$ vs $\left.O \mathrm{R}_{\mathrm{IVW}}=0.90\right), \mathrm{XL}-\mathrm{HDL}-\mathrm{FC}\left(\mathrm{OR}_{\mathrm{MR} \text {-PRESSO }}=0.0002\right.$ vs $\left.\mathrm{OR}_{\mathrm{IVW}}=0.90\right)$ that significant sensitivity results yielded discordant point estimates with IVW. Another six lipoproteins, IDL-FC, IDL-PL, M-LDL-C, S-HDL-P, XL-HDL-C, and XL-HDL-P also showed inconsistent results of weighted median and weighted mode with IVW.

\section{Validation}

In validation, we used UK Biobank dataset to verify the association between IVW-identified metabolites and proxy-AD (Fig 3). Of the 32 identified metabolites, 20 were still significant associated with proxy AD in validation, especially including $A p o B, G p$, and LDL-C, and several different composition of LDL fractions. Among 32 selected metabolites, direction of point estimates in validation were all accordant 
with primary results in IVW except for the citrate $\left(O R_{\text {validation }}=1.029\right)$, which yields reversed effect with risk of $A D\left(O R_{\mathrm{IVW}}=0.83\right)$.

\section{Pathway enrichment analysis}

Our study identified six significant metabolic pathways that were involved in the pathogenesis of $A D$ (Table 2). The most significant metabolic pathway was D-glutamine and D-glutamate metabolism ( $P$ $=1.30 \times 10^{-5}$ ) from the KEGG database. L-glutamine and L-glutamine were involved in this metabolic pathway. Another two metabolic pathways involving two circulating metabolites (i.e., glutamine and

citrate) survived FDR correction, that is, the pathway of alanine, aspartate, and glutamate metabolism ( $P$ $=0.0003)$, and glyoxylate and dicarboxylate metabolism $(P=0.0004)$. We also identified three pathways at the nominal $P<0.05$, including nitrogen metabolism $(P=0.008)$, arginine biosynthesis $(P=0.018)$, and citrate cycle (i.e., tricarboxylic acid $(\mathrm{TCA})$ cycle; $\mathrm{P}=0.026$ ).

\section{Discussion}

By performing a two-sample MR analysis, the present study supports the hypothesis that circulating metabolites levels can be causally corelated to risk of AD. We suggest a significant association between higher levels of $\mathrm{Gp}$ and higher risk of $A D$, and genetically predicted glutamine levels are significantly associated with lower risk of AD. Our results also reinforce the idea that circulating lipid-related metabolites may play a role in the in the pathophysiological process of AD. Particularly, we observed robust evidences of causal effects with respect to $A p o B$, serum-C, three IDL subfractions (i.e., IDL-C, IDL-L, IDL-P), and 13 LDL subfractions (i.e., LDL-C, L-LDL-C, L-LDL-CE, L-LDL-FC, L-LDL-L, L-LDL-P, L-LDL-PL, MLDL-CE, M-LDL-L, M-LDL-P, M-LDL-PL, S-LDL-C, and S-LDL-P) on higher risk of AD.

The measured Gp are mainly a-1-acid glycoprotein (AGP) [12], also called orosomucoid. Gp is an acute phase plasma a-globulin glycoprotein, involving in many activities including modulating immunity, binding and carrying drugs, maintaining the barrier function of capillary, and mediating the sphingolipid metabolism [30-32]. Gp is associated with AD due to its important role in modulating neuroinflammation [33]. Higher levels of plasma AGP were found in patients with cognitive impairment than in normal subjects [33]. Previous meta-analysis has reported that plasma levels of $G p$ were associated with increased risk of dementia and lower cognitive function [8]. These results support our MR findings suggesting a relationship between circulating Gp and AD.

Consistently, a recent published MR study also highlighted the Gp and XL-HDLs as AD causal candidates [34]. This MR analysis limited to 20 metabolites previously reported to be associated with midlife cognition in observational analyses. However, caution should be taken in the interpretation of observational results, as possible unmeasured confounding and reverse causation can result in spurious associations, misdirection of effect, and diluted associations due to lack of precision. It may lead to selection bias to limit the metabolites based on only one observational study [35]. The adoption of 123 
circulating metabolites summary statistics enabled us to detect some potential causalities that had previously been overlooked in MR analysis, such as ApoB, citrate, and glutamine, etc.

$A p o B$ is synthesized in the liver and circulates in the plasma as the major protein component of $L D L$, involving in the transport of cholesterol to peripheral tissues [36]. Previous studies have demonstrated that $A D$ group has significantly higher levels $A p o B$ in serum $[37,38]$ and plasma $[39]$ than that of the control group, especially in AD subjects with APOE $\varepsilon 4$ allele [40]. In AD patients, higher serum levels of $A p o B$ are significantly correlated with higher $A \beta 42$ levels in brain [38]. Additionally, genetic variants in the gene of $A P O B$ are strongly associated with early-onset $A D$ [41], suggesting a link of $A p o B$ to $A D$ risk. However, previous studies of circulating $A p o B$ levels in human are conflicting, with a large population study finding no association between circulating ApoB levels and incident AD [42]. Therefore, our MR results is a significant evidence enhancing the association between circulating $A p o B$ and $A D$ and suggesting it as causality.

Some biological studies have reported coincident evidences with our findings of ApoB. Plasma ApoB was found co-localized with cerebral amyloid plaque in a transgenic mouse AD model [43], and was positively correlated with $\mathrm{Ab}$ plaque abundance in brain [44]. Overexpressing $A P O B$ in a transgenic mouse model induces significant memory impairment and increases $A \beta$ levels compared with wild-type mice, suggesting that increased ApoB levels can contribute to the development of AD-like pathology [45].

Whereas ApoB is involved in LDL-C metabolism and is regarded as a promising link between cholesterol and $A D$ [46], many epidemiological evidences of association between LDL-C and AD are consistent with that of ApoB [38]. Observational studies have indicated that LDL-C levels were significantly increased in $A D$ patients [47-49]. Likewise, Zhou et al. suggest that elevated concentration of LDL-C (>121 mg/dl) may be a potential risk factor for $A D$ [50]. Our MR analysis support these results and suggest a causal effect of high circulating LDL-C levels in increasing risk of AD. Consistent with our findings, another two published MR study also revealed similar effects of LDL-C using different datasets [51, 52], enhancing reliability of the results.

According to the molecular size, LDLs are further categorized as large (L), medium (M), and small (S) LDLs in initial study [12]. Variation in circulating levels and composition of these fractions may have different pathophysiologic significance. Particularly, plasma levels of L-LDL particles were significantly associated with greater cerebral amyloidosis and lower hippocampal volumes independent of LDL-C [53]. Except for LDL-C, our findings suggest that six L-LDL subfractions, four M-LDL subfractions, and two SLDL subfractions can influence the $A D$ risk, but further investigations are needed to fully understand the molecular mechanisms involved.

In the results of observational studies, the effects of serum-C on AD risk were highly heterogeneous [54]; several meta-analyses revealed non-significant effect on AD [55], while other epidemiological studies reported that serum-C levels were significantly increased in AD patients [47-49]. The significance of serum TC differs between mid-life and older adults [56]. Several studies state that high mid-life serum TC levels represent a risk factor for subsequent $A D$ [57], but that there are no detectable differences in serum TC 
levels at older ages [58]. Additionally, except for long-term average serum TC levels, higher TC variability is significantly associated with increased risk of all-cause dementia and AD in the general population, independent of mean TC levels [59]. Thus, the in-coincident results between serum-C studies may be explained by the variations in total cholesterol levels and the disease progression. Taking the advantage of not being affected by unmeasured confounders inherent in observational studies [9], our MR results are more robust, suggesting the high serum-C levels may have a causal effect in increasing AD risk.

Many epidemiological evidences suggest a protective association of circulating HDL cholesterol (HDL-C) levels against $A D$ risk [60]. While we found HDL-C levels are not associated with AD risk with enough power (see Additional file 1: Table S3), concordant with a large population study [42]. In our study, four very large HDL subfractions (i.e., XL-HDL-C, XL-HDL-CE, XL-HDL-FC, and XL-HDL-P) yield inverse effects in $A D$ risk, however, these sensitivity results showed inconsistent effect estimates against IVW. Investigations are needed to further clarify whether the relationship between $H D L$ and AD are causal.

Our study also reported several metabolic pathways that might be involved in the pathogenesis of $A D$, in which the D-glutamine and D-glutamate metabolism has been reported to be associated with $A D[4,61]$. Observational study showed that glutamine concentrations in plasma is positively correlated with that in posterior cingulate cortex [62], which is associated with cognitive impairment in AD [63]. We found consistent results that genetically determined circulating glutamine show a protective effect against AD. Nevertheless, a cohort study found higher glutamine levels were associated with lower cognitive function and higher risk of dementia [8]. Whereas observational studies are prone to reverse causation and confounding bias, an MR analysis with balanced horizontal pleiotropy is more credible [10]. Consistent with our results, a published two-sample MR study came to a similar conclusion of glutamine using a different $A D$ dataset [64]. Furthermore, by conducting a series of rigorous sensitivity, pleiotropy, and validation analyses, our results are more comprehensive and robust. Moreover, there also exist biological evidences of this result. Anderson et al. have observed that reduced glutamine metabolism, reduced TCA activity, and impaired oxidative glutamine metabolism precede amyloid plaque formation in AD mouse model compared to controls [65]. And glutamine is proved to protect against oxidative stress-induced injury that is intimately related to $A D$ in $A D$ mice model [66].

Citrate is key constituent of the TCA cycle, serves as a substrate in the cellular energy metabolism cycle involved in the fatty acid synthesis, glycolysis, and gluconeogenesis [67]. There are very few researches exploring the relationship between citrate and AD. However, our current analysis found a protective effect of citrate in $A D$ risk at significant level. Although additional evidence is needed, it might provide valuable information to help understand the underlying biological mechanisms in the pathogenesis of $A D$.

Our study also has several limitations. First, a general challenge of MR is the persistent possibility of horizontal pleiotropic associations between exposure and outcome. In the present study, we conducted up-to-date analyses to detect and correct the potential pleiotropy. One limitation is that the Q-test and MRPRESSO global test is significant in some metabolites. Nevertheless, MR-PRESSO outlier test was further performed to correct for horizontal pleiotropy and returned an unbiased causal estimate. Second, some 
metabolites yield opposite direction of effect estimates across sensitivity analysis and IVW. It is generally recommended that the emphasis of sensitivity analysis should be laid on the direction of point estimates among the IVW and sensitivity analyses, rather than just the $P$ values. Although this standard ruled out several metabolites from robust results, a serious screening protocol ensure the reliability of our results. For instance, the Gp and glutamine showed the most robust casualty. However, we didn't have enough evidence that those "non-robust" metabolites are not associated with AD. Third, we used a proxy-AD GWAS dataset to verify our analysis. Hence, the phenotypes used for validation were different from that used in primary analysis, resulting in smaller effect sizes. However, the validation is an independent replication analysis for that there is no overlap between primary AD dataset and validation dataset.

Despite these limitations, strengths of the study are notable. Our study provided novel insight by combining metabolomics with genomics to help understand the pathogenesis of AD. The use of two sample MR approach also enabled us to use the very large AD case-control data, giving sufficient power to detect even small effects. And there is no overlapping among exposure and outcome datasets, as is unachievable in many MR studies that may bias effect estimates. Stronger evidence of causal relationships is of great importance because the $A D$ underlying pathophysiological mechanisms are unclear. If these circulating metabolites levels truly reduce $A D$ risk, it would be promising markers for early detection and potential avenues for effective therapeutic intervention in AD.

\section{Conclusions}

In conclusion, our study suggested increased levels of circulating Gp, ApoB, LDL-C, and serum-C were the most robust metabolites that were associated with higher risk of $A D$, whereas glutamine showed the contrary effect. We found strong evidence for causal effects of several different composition of LDL fractions on increased $A D$ risk. The present study provides little evidence that recommending circulating HDL-C would help to prevent AD. Further research is required to decipher the biological pathways underpinning associations.

\section{Abbreviations}

AD, Alzheimer's disease; ADGC, Alzheimer Disease Genetics Consortium; AGP, a-1-acid glycoprotein; Cl, confidence interval; CHARGE, Cohorts for Heart and Aging Research in Genomic Epidemiology Consortium; EADI, The European Alzheimer's Disease Initiative; FDR, false discovery rates; GERAD/PERADES, Genetic and Environmental Risk in Alzheimer's Disease /Defining Genetic, Polygenic and Environmental Risk for Alzheimer's Disease Consortium; GWAS, genome-wide association studies; Gp, glycoprotein acetyls; HDL, high-density lipoprotein; IDL, intermediate-density lipoprotein; KEGG: Kyoto Encyclopedia of Genes and Genomes; LDL, low-density lipoprotein; NOME, NO Measurement Error; OR, odds ratio; SD, standard deviation; SNP, single nucleotide polymorphism; IGAP, International Genomics of Alzheimer's Project; IV, instrumental variable; IVW, inverse variance weighting; MBE, mode-based estimate; MR, Mendelian randomization; MR-PRESSO, Mendelian randomization pleiotropy residual sum and outlier; serum-C, serum total cholesterol; TCA, tricarboxylic acid. 


\section{Declarations}

\section{Ethics approval and consent to participate}

All data sources used in this MR study received approval from an ethics standards committee on human experimentation and obtained informed consent from all participants.

\section{Consent for publication}

Not applicable.

\section{Availability of data and materials}

All the data used in this study can be acquired from the original genome-wide association studies that are mentioned in the text. Any other data generated in the analysis process can be requested from the corresponding author.

\section{Competing interests}

The authors declare that they have no competing interests.

\section{Funding}

This study was supported by grants from the National Natural Science Foundation of China (91849126), the National Key R\&D Program of China (2018YFC1314702), Shanghai Municipal Science and Technology Major Project (No.2018SHZDZX03) and ZHANGJIANG LAB, Tianqiao and Chrissy Chen Institute, and the State Key Laboratory of Neurobiology and Frontiers Center for Brain Science of Ministry of Education, Fudan University. The funding bodies had no role in the design of the study and collection, analysis, and interpretation of data, or in writing the manuscript.

\section{Authors' contributions}

Jin-Tai Yu had full access to all the data in the study and takes responsibility for the integrity of the data and the accuracy of the data analysis.

Concept and design: Jin-Tai Yu.

Acquisition, analysis, or interpretation of data: All authors.

Drafting of the manuscript: Shu-Yi Huang

Critical revision of the manuscript for important intellectual content: All authors.

Statistical analysis: Shu-Yi Huang

Obtained funding: Jin-Tai Yu. 
Administrative, technical, or material support: Jin-Tai Yu

Supervision: Jin-Tai Yu.

All authors read and approved the final manuscript.

\section{Acknowledgments}

This work was made possible by the generous sharing of GWAS summary statistics. We thank the participants, researchers, and staff associated with the many other studies from which we used data for this report. We thank the UK Biobank for providing summary statistics for these analyses. We also thank the IGAP for providing summary results data for these analyses. The investigators within IGAP contributed to the design and implementation of IGAP and/or provided data but did not participate in analysis or writing of this report. IGAP was made possible by the generous participation of the control subjects, the patients, and their families. The i-Select chips were funded by the French National Foundation on Alzheimer's disease and related disorders. EADI was supported by the LABEX DISTALZ grant, Inserm, Institut Pasteur de Lille, Université de Lille 2 and the Lille University Hospital. GERAD was supported by the Medical Research Council (Grant $n^{\circ}$ 503480), Alzheimer's Research UK (Grant $n^{\circ}$ 503176), the Wellcome Trust (Grant $n^{\circ} 082604 / 2 / 07 / Z$ ) and German Federal Ministry of Education and Research (BMBF): Competence Network Dementia (CND) grant n 01GI0102, 01GI0711, $01 \mathrm{GI0420.}$ CHARGE was partly supported by the NIH/NIA grant R01 AG033193 and the NIA AG081220 and AGES contract N01-AG-12100, the NHLBI grant R01 HL105756, the Icelandic Heart Association, and the Erasmus Medical Center and Erasmus University. ADGC was supported by the NIH/NIA grants: U01 AG032984, U24 AG021886, U01 AG016976, and the Alzheimer's Association grant ADGC-10-196728.

\section{References}

1. Winblad B, Amouyel P, Andrieu S, Ballard C, Brayne C, Brodaty H, Cedazo-Minguez A, Dubois B, Edvardsson $\mathrm{D}$, Feldman $\mathrm{H}$, et al: Defeating Alzheimer's disease and other dementias: a priority for European science and society. The Lancet Neurology 2016, 15:455-532.

2. Scheltens P, Blennow K, Breteler MMB, de Strooper B, Frisoni GB, Salloway S, Van der Flier WM: Alzheimer's disease. The Lancet 2016, 388:505-517.

3. Wilkins JM, Trushina E: Application of Metabolomics in Alzheimer's Disease. Front Neuro/2017, 8:719.

4. Cui M, Jiang Y, Zhao Q, Zhu Z, Liang X, Zhang K, Wu W, Dong Q, An Y, Tang H, et al: Metabolomics and incident dementia in older Chinese adults: The Shanghai Aging Study. Alzheimers Dement 2020, 16:779-788.

5. Varma VR, Oommen AM, Varma S, Casanova R, An Y, Andrews RM, O'Brien R, Pletnikova O, Troncoso $\mathrm{JC}$, Toledo J, et al: Brain and blood metabolite signatures of pathology and progression in Alzheimer 
disease: A targeted metabolomics study. PLoS medicine 2018, 15:e1002482.

6. Huo Z, Yu L, Yang J, Zhu Y, Bennett DA, Zhao J: Brain and blood metabolome for Alzheimer's dementia: findings from a targeted metabolomics analysis. Neurobiology of aging 2020, 86:123-133.

7. Ibáñez C, Cifuentes A, Simó C: Recent advances and applications of metabolomics to investigate neurodegenerative diseases. Int Rev Neurobiol 2015, 122:95-132.

8. van der Lee SJ, Teunissen CE, Pool R, Shipley MJ, Teumer A, Chouraki V, Melo van Lent D, Tynkkynen J, Fischer K, Hernesniemi J, et al: Circulating metabolites and general cognitive ability and dementia: Evidence from 11 cohort studies. Alzheimers Dement 2018, 14:707-722.

9. Davies NM, Holmes MV, Davey Smith G: Reading Mendelian randomisation studies: a guide, glossary, and checklist for clinicians. BMJ 2018, 362:k601.

10. Emdin CA, Khera AV, Kathiresan S: Mendelian Randomization. Jama 2017, 318:1925-1926.

11. Lawlor DA: Commentary: Two-sample Mendelian randomization: opportunities and challenges. Int $J$ Epidemiol 2016, 45:908-915.

12. Kettunen J, Demirkan A, Würtz P, Draisma HH, Haller T, Rawal R, Vaarhorst A, Kangas AJ, Lyytikäinen LP, Pirinen $M$, et al: Genome-wide study for circulating metabolites identifies 62 loci and reveals novel systemic effects of LPA. Nat Commun 2016, 7:11122.

13. Hagenbeek FA, Pool R, van Dongen J, Draisma HHM, Jan Hottenga J, Willemsen G, Abdellaoui A, Fedko IO, den Braber A, Visser PJ, et al: Heritability estimates for 361 blood metabolites across 40 genome-wide association studies. Nat Commun 2020, 11:39.

14. Wishart DS, Feunang YD, Marcu A, Guo AC, Liang K, Vázquez-Fresno R, Sajed T, Johnson D, Li C, Karu N, et al: HMDB 4.0: the human metabolome database for 2018. Nucleic Acids Res 2018, 46:D608d617.

15. Pierce BL, Ahsan H, Vanderweele TJ: Power and instrument strength requirements for Mendelian randomization studies using multiple genetic variants. Int J Epidemiol 2011, 40:740-752.

16. Burgess S: Sample size and power calculations in Mendelian randomization with a single instrumental variable and a binary outcome. Int J Epidemiol 2014, 43:922-929.

17. Kunkle BW, Grenier-Boley B, Sims R, Bis JC, Damotte V, Naj AC, Boland A, Vronskaya M, van der Lee SJ, Amlie-Wolf A, et al: Genetic meta-analysis of diagnosed Alzheimer's disease identifies new risk loci and implicates A 3 , tau, immunity and lipid processing. Nat Genet 2019, 51:414-430.

18. Sudlow C, Gallacher J, Allen N, Beral V, Burton P, Danesh J, Downey P, Elliott P, Green J, Landray M, et al: UK biobank: an open access resource for identifying the causes of a wide range of complex diseases of middle and old age. PLoS medicine 2015, 12:e1001779.

19. Marioni RE, Harris SE, Zhang Q, McRae AF, Hagenaars SP, Hill WD, Davies G, Ritchie CW, Gale CR, Starr JM, et al: GWAS on family history of Alzheimer's disease. Translational psychiatry 2018, 8:99.

20. Liu JZ, Erlich Y, Pickrell JK: Case-control association mapping by proxy using family history of disease. Nat Genet 2017, 49:325-331. 
21. Bowden J, Del Greco MF, Minelli C, Davey Smith G, Sheehan N, Thompson J: A framework for the investigation of pleiotropy in two-sample summary data Mendelian randomization. Statistics in medicine 2017, 36:1783-1802.

22. Bowden J, Davey Smith G, Haycock PC, Burgess S: Consistent Estimation in Mendelian Randomization with Some Invalid Instruments Using a Weighted Median Estimator. Genetic epidemiology 2016, 40:304-314.

23. Hartwig FP, Davey Smith G, Bowden J: Robust inference in summary data Mendelian randomization via the zero modal pleiotropy assumption. Int J Epidemiol 2017, 46:1985-1998.

24. Bowden J, Davey Smith G, Burgess S: Mendelian randomization with invalid instruments: effect estimation and bias detection through Egger regression. Int J Epidemiol 2015, 44:512-525.

25. Verbanck M, Chen CY, Neale B, Do R: Detection of widespread horizontal pleiotropy in causal relationships inferred from Mendelian randomization between complex traits and diseases. Nat Genet 2018, 50:693-698.

26. Bowden J, Del Greco MF, Minelli C, Davey Smith G, Sheehan NA, Thompson JR: Assessing the suitability of summary data for two-sample Mendelian randomization analyses using MR-Egger regression: the role of the 12 statistic. Int J Epidemiol 2016, 45:1961-1974.

27. Hemani G, Zheng J, Elsworth B, Wade KH, Haberland V, Baird D, Laurin C, Burgess S, Bowden J, Langdon R, et al: The MR-Base platform supports systematic causal inference across the human phenome. Elife 2018, 7.

28. Rasooly D, Patel CJ: Conducting a Reproducible Mendelian Randomization Analysis Using the R Analytic Statistical Environment. Curr Protoc Hum Genet 2019, 101:e82.

29. Kanehisa M, Goto S, Sato Y, Furumichi M, Tanabe M: KEGG for integration and interpretation of largescale molecular data sets. Nucleic Acids Res 2012, 40:D109-114.

30. Duprez DA, Jacobs DR, Jr.: GlycA, a composite low-grade inflammatory marker, predicts mortality: prime time for utilization? J Intern Med 2019, 286:610-612.

31. Gruppen EG, Kunutsor SK, Kieneker LM, van der Vegt B, Connelly MA, de Bock GH, Gansevoort RT, Bakker SJL, Dullaart RPF: GlycA, a novel pro-inflammatory glycoprotein biomarker is associated with mortality: results from the PREVEND study and meta-analysis. J Intern Med 2019, 286:596-609.

32. Luo Z, Lei H, Sun Y, Liu X, Su DF: Orosomucoid, an acute response protein with multiple modulating activities. J Physiol Biochem 2015, 71:329-340.

33. Jo M, Kim JH, Song GJ, Seo M, Hwang EM, Suk K: Astrocytic Orosomucoid-2 Modulates Microglial Activation and Neuroinflammation. J Neurosci 2017, 37:2878-2894.

34. Lord J, Jermy B, Green R, Wong A, Xu J, Legido-Quigley C, Dobson R, Richards M, Proitsi P: Mendelian randomization identifies blood metabolites previously linked to midlife cognition as causal candidates in Alzheimer's disease. Proceedings of the National Academy of Sciences of the United States of America 2021, 118.

35. Proitsi P, Kuh D, Wong A, Maddock J, Bendayan R, Wulaningsih W, Hardy R, Richards M: Lifetime cognition and late midlife blood metabolites: findings from a British birth cohort. Translational 
psychiatry 2018, 8:203.

36. Chan L: Apolipoprotein B, the major protein component of triglyceride-rich and low density lipoproteins. J Biol Chem 1992, 267:25621-25624.

37. Caramelli P, Nitrini R, Maranhão R, Lourenço AC, Damasceno MC, Vinagre C, Caramelli B: Increased apolipoprotein B serum concentration in Alzheimer's disease. Acta Neurol Scand 1999, 100:61-63.

38. Kuo YM, Emmerling MR, Bisgaier CL, Essenburg AD, Lampert HC, Drumm D, Roher AE: Elevated lowdensity lipoprotein in Alzheimer's disease correlates with brain abeta 1-42 levels. Biochem Biophys Res Commun 1998, 252:711-715.

39. Raygani AV, Rahimi Z, Kharazi H, Tavilani H, Pourmotabbed T: Association between apolipoprotein E polymorphism and serum lipid and apolipoprotein levels with Alzheimer's disease. Neurosci Lett 2006, 408:68-72.

40. Song F, Poljak A, Crawford J, Kochan NA, Wen W, Cameron B, Lux O, Brodaty H, Mather K, Smythe GA, Sachdev PS: Plasma apolipoprotein levels are associated with cognitive status and decline in a community cohort of older individuals. PLoS One 2012, 7:e34078.

41. Wingo TS, Cutler DJ, Wingo AP, Le NA, Rabinovici GD, Miller BL, Lah JJ, Levey Al: Association of Early-Onset Alzheimer Disease With Elevated Low-Density Lipoprotein Cholesterol Levels and Rare Genetic Coding Variants of APOB. JAMA Neurol 2019, 76:809-817.

42. Tynkkynen J, Hernesniemi JA, Laatikainen T, Havulinna AS, Sundvall J, Leiviskä J, Salo P, Salomaa $\mathrm{V}$ : Apolipoproteins and HDL cholesterol do not associate with the risk of future dementia and Alzheimer's disease: the National Finnish population study (FINRISK). Age (Dordr) 2016, 38:465-473.

43. Takechi R, Galloway S, Pallebage-Gamarallage M, Wellington C, Johnsen R, Mamo JC: Threedimensional colocalization analysis of plasma-derived apolipoprotein $B$ with amyloid plaques in APP/PS1 transgenic mice. Histochem Cell Biol 2009, 131:661-666.

44. Takechi R, Galloway S, Pallebage-Gamarallage MM, Wellington CL, Johnsen RD, Dhaliwal SS, Mamo JC: Differential effects of dietary fatty acids on the cerebral distribution of plasma-derived apo $B$ lipoproteins with amyloid-beta. Br J Nutr 2010, 103:652-662.

45. Löffler T, Flunkert S, Havas D, Sántha M, Hutter-Paier B, Steyrer E, Windisch M: Impact of ApoB-100 expression on cognition and brain pathology in wild-type and hAPPsl mice. Neurobiology of aging 2013, 34:2379-2388.

46. Ishii M: Apolipoprotein B as a New Link Between Cholesterol and Alzheimer Disease. JAMA Neurol 2019, 76:751-753.

47. Liu Y, Zhong X, Shen J, Jiao L, Tong J, Zhao W, Du K, Gong S, Liu M, Wei M: Elevated serum TC and LDL-C levels in Alzheimer's disease and mild cognitive impairment: A meta-analysis study. Brain Res 2020, 1727:146554.

48. Wu Y, Wang Z, Jia X, Zhang H, Zhang H, Li J, Zhang K: Prediction of Alzheimer's disease with serum lipid levels in Asian individuals: a meta-analysis. Biomarkers 2019, 24:341-351.

49. Wang $P$, Zhang $H$, Wang $Y$, Zhang $M$, Zhou Y: Plasma cholesterol in Alzheimer's disease and frontotemporal dementia. Trans/ Neurosci 2020, 11:116-123. 
50. Zhou Z, Liang Y, Zhang X, Xu J, Lin J, Zhang R, Kang K, Liu C, Zhao C, Zhao M: Low-Density Lipoprotein Cholesterol and Alzheimer's Disease: A Systematic Review and Meta-Analysis. Frontiers in aging neuroscience 2020, 12:5.

51. Østergaard SD, Mukherjee S, Sharp SJ, Proitsi P, Lotta LA, Day F, Perry JR, Boehme KL, Walter S, Kauwe JS, et al: Associations between Potentially Modifiable Risk Factors and Alzheimer Disease: A Mendelian Randomization Study. PLoS medicine 2015, 12:e1001841; discussion e1001841.

52. Benn M, Nordestgaard BG, Frikke-Schmidt R, Tybjærg-Hansen A: Low LDL cholesterol, PCSK9 and HMGCR genetic variation, and risk of Alzheimer's disease and Parkinson's disease: Mendelian randomisation study. Bmj 2017, 357:j1648.

53. Lee S, Parekh T, King SM, Reed B, Chui HC, Krauss RM, Yassine HN: Low-Density Lipoprotein Particle Size Subfractions and Cerebral Amyloidosis. Journal of Alzheimer's disease : JAD 2019, 68:983-990.

54. Sáiz-Vazquez O, Puente-Martínez A, Ubillos-Landa S, Pacheco-Bonrostro J, Santabárbara J: Cholesterol and Alzheimer's Disease Risk: A Meta-Meta-Analysis. Brain sciences 2020, 10.

55. Xu W, Tan L, Wang HF, Jiang T, Tan MS, Tan L, Zhao QF, Li JQ, Wang J, Yu JT: Meta-analysis of modifiable risk factors for Alzheimer's disease. Journal of neurology, neurosurgery, and psychiatry 2015, 86:1299-1306.

56. Solomon A, Kåreholt I, Ngandu T, Winblad B, Nissinen A, Tuomilehto J, Soininen H, Kivipelto M: Serum cholesterol changes after midlife and late-life cognition: twenty-one-year follow-up study. Neurology 2007, 68:751-756.

57. Kivipelto M, Helkala EL, Laakso MP, Hänninen T, Hallikainen M, Alhainen K, livonen S, Mannermaa A, Tuomilehto J, Nissinen A, Soininen H: Apolipoprotein E epsilon4 allele, elevated midlife total cholesterol level, and high midlife systolic blood pressure are independent risk factors for late-life Alzheimer disease. Annals of internal medicine 2002, 137:149-155.

58. Tan ZS, Seshadri S, Beiser A, Wilson PW, Kiel DP, Tocco M, D'Agostino RB, Wolf PA: Plasma total cholesterol level as a risk factor for Alzheimer disease: the Framingham Study. Arch Intern Med 2003, 163:1053-1057.

59. Chung HS, Lee JS, Kim JA, Roh E, Lee YB, Hong SH, Kim NH, Yoo HJ, Seo JA, Kim SG, et al: Variability in Total Cholesterol Concentration Is Associated With the Risk of Dementia: A Nationwide Population-Based Cohort Study. Front Neuro/ 2019, 10:441.

60. Pedrini S, Chatterjee $P$, Hone E, Martins RN: High-density lipoprotein-related cholesterol metabolism in Alzheimer's disease. J Neurochem 2020.

61. Huang D, Liu D, Yin J, Qian T, Shrestha S, Ni H: Glutamate-glutamine and GABA in brain of normal aged and patients with cognitive impairment. Eur Radiol 2017, 27:2698-2705.

62. Takado Y, Sato N, Kanbe Y, Tomiyasu M, Xin L, Near J, Yoshikawa K, Sahara N, Higashi T, Suhara T, et al: Association between Brain and Plasma Glutamine Levels in Healthy Young Subjects Investigated by MRS and LC/MS. Nutrients 2019, 11.

63. Uddin LQ, Kelly AM, Biswal BB, Castellanos FX, Milham MP: Functional connectivity of default mode network components: correlation, anticorrelation, and causality. Hum Brain Mapp 2009, 30:625-637. 
64. Adams CD: Circulating Glutamine and Alzheimer's Disease: A Mendelian Randomization Study. Clin Interv Aging 2020, 15:185-193.

65. Andersen JV, Christensen SK, Aldana BI, Nissen JD, Tanila H, Waagepetersen HS: Alterations in Cerebral Cortical Glucose and Glutamine Metabolism Precedes Amyloid Plaques in the APPswe/PSEN1dE9 Mouse Model of Alzheimer's Disease. Neurochem Res 2017, 42:1589-1598.

66. Wang Y, Wang Q, Li J, Lu G, Liu Z: Glutamine Improves Oxidative Stress through the Wnt3a/ $\beta$-Catenin Signaling Pathway in Alzheimer's Disease In Vitro and In Vivo. Biomed Res Int 2019, 2019:4690280.

67. Westergaard N, Waagepetersen HS, Belhage B, Schousboe A: Citrate, a Ubiquitous Key Metabolite with Regulatory Function in the CNS. Neurochem Res 2017, 42:1583-1588.

\section{Tables}

Table 1. Pleiotropy and heterogeneity analyses for the association between metabolites and Alzheimer's disease in primary analysis 
Metabolites

MR-Egger intercept Pvalue
$Q$ test $P$ value
MR-PRESSO global Corrected MR-PRESSO test P-value
$\mathrm{OR}$

$(95 \% \mathrm{Cl})$
P-value

\section{Protein}

ApoB

0.80

$<0.001$

$<0.001$

2.20

(1.66,

$2.74 \times 10^{-04}$

2.92)

$\begin{array}{lll}\text { Glycoprotein acetyls } & 0.83 & 0.12\end{array}$

\section{Carboxylic acids}

and derivatives

\begin{tabular}{llll} 
Citrate & 0.48 & 0.93 & 0.96 \\
\hline Glutamine & 0.92 & 1.00 & 0.88
\end{tabular}

\section{Lipoprotein}

IDL-C

0.53

$<0.001<0.001$

1.72

(1.48,

$1.65 \times 10^{-07}$

1.99)

'DL-FC

0.27

$<0.001<0.001$

1.08

0.074

(0.99,

1.17)

IDL-L

0.26

$<0.001$

$<0.001$

1.24

0.002

(1.09,

1.40)

IDL-P

0.33

$<0.001<0.001$

1.35

(1.16,

1.57)

'DL-PL

0.83

$<0.001<0.001$

1.69

$(1.43$

1.98)

LDL-C $\quad 0.44 \quad<0.001<0.001$

1.59

(1.40,

$2.83 \times 10^{-08}$

1.81)

L-LDL-C

0.41

$<0.001<0.001$

1.67

(1.46,

1.91)

L-LDL-CE

0.73

$<0.001$

$<0.001$

1.69

(1.49,

1.91)

L-LDL-FC

0.41

$<0.001<0.001$

1.69

(1.47,

$6.58 \times 10^{-08}$

$1.19 \times 10^{-06}$

$7.22 \times 10^{-04}$ 


\begin{tabular}{|c|c|c|c|c|c|}
\hline L-LDL-L & 0.51 & $<0.001$ & $<0.001$ & $\begin{array}{l}1.61 \\
(1.40 \\
1.85)\end{array}$ & $1.77 \times 10^{-07}$ \\
\hline L-LDL-P & 0.34 & $<0.001$ & $<0.001$ & $\begin{array}{l}1.68 \\
(1.47 \\
1.92)\end{array}$ & $2.38 \times 10^{-08}$ \\
\hline L-LDL-PL & 0.34 & $<0.001$ & $<0.001$ & $\begin{array}{l}1.64 \\
(1.45, \\
1.84)\end{array}$ & $3.45 \times 10^{-09}$ \\
\hline$M_{-L D L-C}$ & 0.42 & $<0.001$ & $<0.001$ & $\begin{array}{l}1.81 \\
(1.57 \\
2.09)\end{array}$ & $4.10 \times 10^{-08}$ \\
\hline M-LDL-CE & 0.40 & $<0.001$ & $<0.001$ & $\begin{array}{l}1.74 \\
(1.50 \\
2.01)\end{array}$ & $8.98 \times 10^{-08}$ \\
\hline$M_{-L D L-L}$ & 0.42 & $<0.001$ & $<0.001$ & $\begin{array}{l}1.89 \\
(1.62, \\
2.21)\end{array}$ & $1.45 \times 10^{-07}$ \\
\hline M-LDL-P & 0.17 & $<0.001$ & $<0.001$ & $\begin{array}{l}1.78 \\
(1.52, \\
2.09)\end{array}$ & $3.32 \times 10^{-07}$ \\
\hline$M_{-L D L-P L}$ & 0.33 & $<0.001$ & $<0.001$ & $\begin{array}{l}1.91 \\
(1.59 \\
2.30)\end{array}$ & $3.53 \times 10^{-06}$ \\
\hline S-LDL-C & 0.82 & $<0.001$ & $<0.001$ & $\begin{array}{l}1.96 \\
(1.61 \\
2.39)\end{array}$ & $4.07 \times 10^{-06}$ \\
\hline$S_{-L D L-L}$ & 0.44 & $<0.001$ & $<0.001$ & $\begin{array}{l}2.09 \\
(1.62 \\
2.69)\end{array}$ & $5.46 \times 10^{-05}$ \\
\hline S-LDL-P & 0.14 & $<0.001$ & $<0.001$ & $\begin{array}{l}2.09 \\
(1.62, \\
2.70)\end{array}$ & $4.38 \times 10^{-05}$ \\
\hline$S_{-H D L-P}$ & 0.19 & 0.45 & $<0.001$ & $\begin{array}{l}1.93 \\
(1.60 \\
2.33)\end{array}$ & $2.62 \times 10^{-06}$ \\
\hline XL-HDL-C & 0.81 & 0.11 & 0.11 & - & - \\
\hline XL-HDL-CE & 0.47 & 0.12 & 0.13 & - & - \\
\hline XL-HDL-FC & 0.72 & 0.09 & $<0.001$ & $\begin{array}{l}1.64 \\
(1.45, \\
1.84)\end{array}$ & $3.45 \times 10^{-09}$ \\
\hline$x_{L-H D L-P}$ & 0.96 & $<0.001$ & $<0.001$ & $\begin{array}{l}0.91 \\
(0.86, \\
0.98)\end{array}$ & 0.012 \\
\hline
\end{tabular}



XS-VLDL-PL
0.13
$<0.001<0.001$
1.03
$(0.97$
1.09)
0.396

\section{Steroids and steroid derivatives}

\begin{tabular}{llllll} 
Serum-C & 0.78 & $<0.001$ & 0.60 & - & - \\
\hline $\begin{array}{l}\text { Esterified } \\
\text { cholesterol }\end{array}$ & 0.48 & $<0.001$ & $<0.001$ & 2.18 & 0.004 \\
& & & & $(1.54$, & \\
\end{tabular}

$\mathrm{Cl}$, confidence interval; MR-PRESSO, Mendelian randomization pleiotropy residual sum and outlier; OR, odds ratio.

Table 2. Significant metabolic pathways involved in the pathogenesis of Alzheimer's disease.

\begin{tabular}{lllll}
\hline Metabolic pathway & Metabolites involved & P value & FDR & Database \\
\hline $\begin{array}{l}\text { D-Glutamine and D-glutamate } \\
\text { metabolism }\end{array}$ & $\begin{array}{l}\text { L-Glutamine; L- } \\
\text { Glutamine }\end{array}$ & $1.30 \times 10^{-05}$ & 0.001 & KEGG \\
\hline $\begin{array}{l}\text { Alanine, aspartate and glutamate } \\
\text { metabolism }\end{array}$ & $\begin{array}{l}\text { L-Glutamine; Citric } \\
\text { acid }\end{array}$ & 0.0003 & 0.012 & KEGG \\
\hline $\begin{array}{l}\text { Glyoxylate and dicarboxylate } \\
\text { metabolism }\end{array}$ & $\begin{array}{l}\text { L-Glutamine; Citric } \\
\text { acid }\end{array}$ & 0.0004 & 0.012 & KEGG \\
\hline Nitrogen metabolism & L-Glutamine & 0.0079 & 0.165 & KEGG \\
\hline Arginine biosynthesis & L-Glutamine & 0.0183 & 0.308 & KEGG \\
\hline Citrate cycle (TCA cycle) & Citric acid & 0.0261 & 0.366 & KEGG
\end{tabular}

FDR, false discovery rates; TCA, tricarboxylic acid.

\section{Figures}


GWAS for 123 circulating metabolic traits

Up to 24,925 individuals from 14 European cohorts

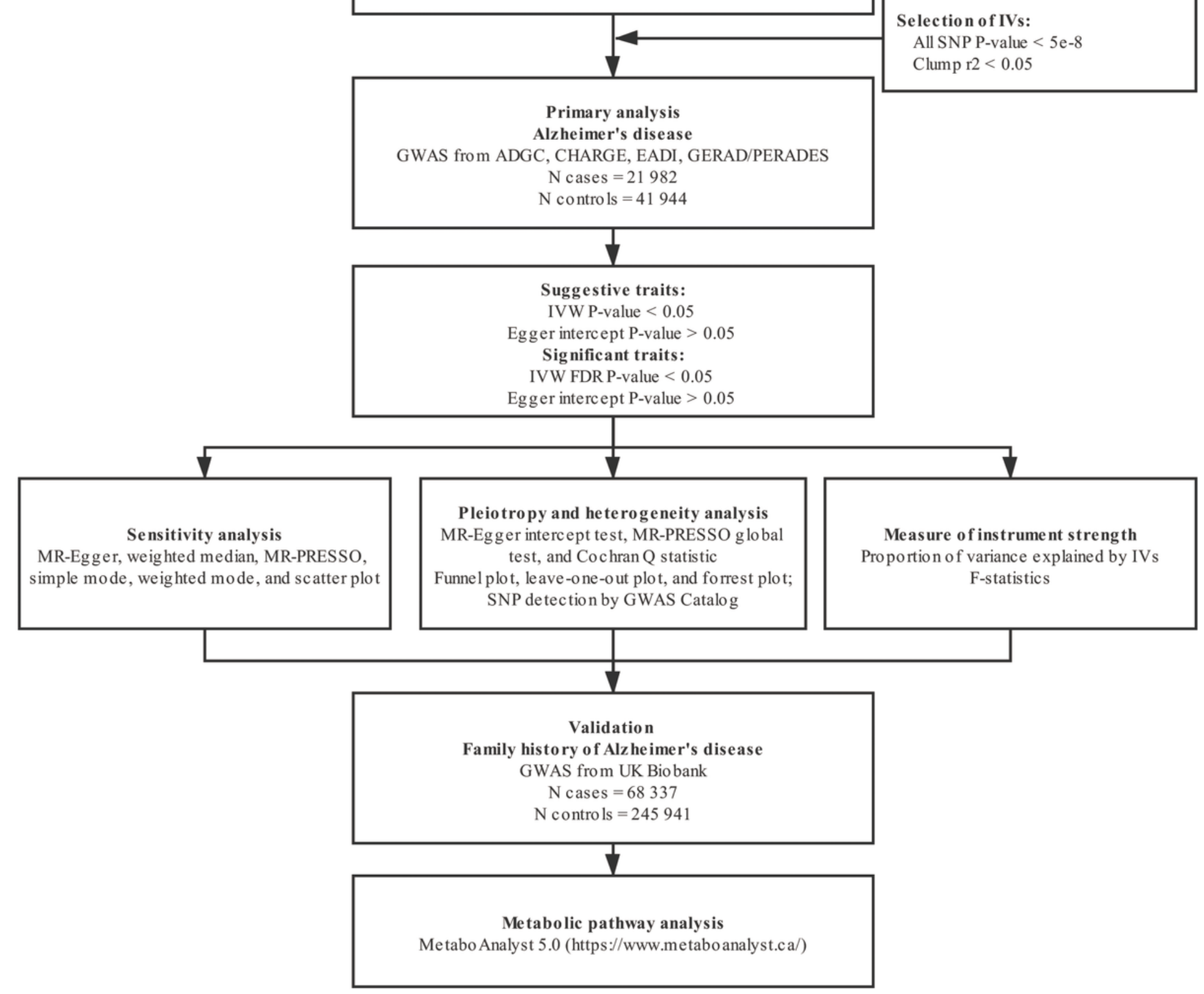

\section{Figure 1}

A flow diagram for the process for identifying genetic variants in the Mendelian randomization analysis ADGC, Alzheimer Disease Genetics Consortium; CHARGE, Cohorts for Heart and Aging Research in Genomic Epidemiology Consortium; EADI, The European Alzheimer's Disease Initiative; GERAD/PERADES, Genetic and Environmental Risk in Alzheimer's Disease /Defining Genetic, Polygenic and Environmental Risk for Alzheimer's Disease Consortium; GWAS, genome-wide association studies; N, number; SNP, single nucleotide polymorphism; IV, instrumental variable; IVW, inverse variance weighting; MR-PRESSO, Mendelian randomization pleiotropy residual sum and outlier. 


\begin{tabular}{|c|c|c|c|c|c|}
\hline Metabolites & SNPs & OR $(95 \% \mathrm{CI})$ & & P-value & FDR \\
\hline \multicolumn{6}{|l|}{ Protein } \\
\hline ApoB & 29 & $3.18(1.52,6.66)$ & $\longmapsto \longrightarrow$ & 0.0022 & 0.014 \\
\hline Glycoprotein acetyls & 12 & $1.21(1.05,1.39)$ & $1 \mapsto-1$ & 0.0093 & 0.043 \\
\hline \multicolumn{6}{|c|}{ Carboxylic acids and derivatives } \\
\hline Citrate & 8 & $0.83(0.73,0.95) \quad 1-1$ & & 0.0055 & 0.029 \\
\hline Glutamine & 6 & $0.81(0.71,0.92) \vdash \bullet$ & & 0.0011 & 0.008 \\
\hline \multicolumn{6}{|l|}{ Lipoprotein } \\
\hline IDL-C & 41 & $2.16(1.31,3.56)$ & $\longmapsto$ & 0.0026 & 0.016 \\
\hline IDL-FC & 43 & $1.46(1.20,1.78)$ & $\mapsto-1$ & 0.0001 & 0.006 \\
\hline IDL-L & 45 & $2.05(1.25,3.35)$ & $\longmapsto$ & 0.0042 & 0.023 \\
\hline IDL-P & 42 & $2.11(1.24,3.57)$ & $\longmapsto$ & 0.0057 & 0.029 \\
\hline IDL-PL & 46 & $2.14(1.31,3.50)$ & $\longmapsto \longrightarrow$ & 0.0023 & 0.014 \\
\hline LDL-C & 52 & $2.34(1.53,3.57)$ & $\longmapsto$ & 0.0001 & 0.01 \\
\hline L-LDL-C & 52 & $2.28(1.47,3.52)$ & $\longmapsto$ & 0.0002 & 0.004 \\
\hline L-LDL-CE & 49 & $2.31(1.47,3.62)$ & $\longmapsto$ & 0.0003 & 0.003 \\
\hline L-LDL-FC & 49 & $2.29(1.46,3.57)$ & $\longmapsto$ & 0.0003 & 0.003 \\
\hline L-LDL-L & 50 & $2.27(1.46,3.54)$ & $\longmapsto$ & 0.0003 & 0.004 \\
\hline L-LDL-P & 51 & $2.28(1.46,3.55)$ & $\longmapsto$ & 0.0003 & 0.003 \\
\hline L-LDL-PL & 50 & $2.36(1.49,3.72)$ & $\longmapsto-1$ & 0.0002 & 0.004 \\
\hline M-LDL-C & 48 & $2.46(1.55,3.90)$ & $\longmapsto$ & 0.0001 & 0.008 \\
\hline M-LDL-CE & 49 & $2.37(1.50,3.75)$ & $\longmapsto$ & 0.0002 & 0.004 \\
\hline M-LDL-L & 48 & $2.44(1.54,3.88)$ & $\longmapsto$ & 0.0002 & 0.004 \\
\hline M-LDL-P & 46 & $2.32(1.46,3.69)$ & $\longmapsto$ & 0.0004 & 0.003 \\
\hline M-LDL-PL & 36 & $2.69(1.51,4.77)$ & $\longmapsto$ & 0.0007 & 0.006 \\
\hline S-LDL-C & 39 & $2.98(1.69,5.23)$ & $\longmapsto$ & 0.0001 & 0.004 \\
\hline S-LDL-L & 39 & $2.69(1.56,4.63)$ & $\longmapsto$ & 0.0004 & 0.003 \\
\hline S-LDL-P & 34 & $2.87(1.52,5.40)$ & $\longmapsto$ & 0.0011 & 0.008 \\
\hline S-HDL-P & 7 & $1.12(1.01,1.25)$ & -1 & 0.0402 & 0.152 \\
\hline XL-HDL-C & 17 & $0.89(0.81,0.98)$ & & 0.0128 & 0.055 \\
\hline XL-HDL-CE & 17 & $0.90(0.82,0.99)$ & & 0.0332 & 0.134 \\
\hline XL-HDL-FC & 22 & $0.90(0.83,0.98)$ & & 0.0108 & 0.048 \\
\hline XL-HDL-P & 23 & $0.90(0.82,0.99)$ & & 0.0274 & 0.114 \\
\hline XS-VLDL-PL & 36 & $1.49(1.11,2.02)$ & $\longmapsto$ & 0.0087 & 0.042 \\
\hline \multicolumn{6}{|c|}{ Steroids and steroid derivatives } \\
\hline Serum-C & 36 & $2.73(1.41,5.30)$ & $\longmapsto$ & 0.003 & 0.017 \\
\hline \multirow[t]{2}{*}{ Esterified cholesterol } & 24 & $2.42(1.07,5.48)$ & $\longmapsto \longrightarrow$ & 0.0345 & 0.135 \\
\hline & & $\begin{array}{r}\Gamma \\
0.70\end{array}$ & $\begin{array}{ll}7 & 1 \\
5.0 & 7.0\end{array}$ & & \\
\hline
\end{tabular}

\section{Figure 2}

Mendelian randomization results for the circulating metabolites and the risks they pose in Alzheimer's disease AD, Alzheimer's disease; $\mathrm{Cl}$, confidence interval; FDR, false discovery rates; SD, standard deviation; SNPs, single nucleotide polymorphisms; OR, odds ratio. 


\section{Protein}

ApoB

Glycoprotein acetyls

Carboxylic acids and derivatives

Citrate

Glutamine

Lipoprotein

IDL-C

IDL-FC

IDL-L

IDL-P

IDL-PL

LDL-C

L-LDL-C

L-LDL-CE

L-LDL-FC

L-LDL-L

L-LDL-P

L-LDL-PL

M-LDL-C

M-LDL-CE

M-LDL-L

M-LDL-P

M-LDL-PL

S-LDL-C

S-LDL-L

S-LDL-P

S-HDL-P

XL-HDL-C

XL-HDL-CE

XL-HDL-FC

XL-HDL-P

XS-VLDL-PL

Steroids and steroid derivatives

Serum-C

Esterified cholesterol

$\begin{array}{cc}26 & 1.31(1,1.72) \\ 12 & 1.05(1.01,1.09)\end{array}$

$8 \quad 1.03(0.98,1.08)$

$6 \quad 0.93(0.85,1.01)$

$35 \quad 1.18(1,1.4)$

$37 \quad 1.11(1.04,1.18)$

$40 \quad 1.17(0.99,1.38)$

$37 \quad 1.18(0.99,1.41)$

$41 \quad 1.19(1.01,1.41)$

$48 \quad 1.18(1.03,1.34)$

$48 \quad 1.17(1.02,1.34)$

$45 \quad 1.18(1.02,1.35)$

$46 \quad 1.17(1.02,1.34)$

$46 \quad 1.17(1.02,1.34)$

$47 \quad 1.17(1.02,1.34)$

$46 \quad 1.18(1.02,1.35)$

$45 \quad 1.18(1.02,1.36)$

$46 \quad 1.17(1.02,1.34)$

$45 \quad 1.17(1.02,1.35)$

$43 \quad 1.17(1.01,1.35)$

$33 \quad 1.23(1.02,1.49)$

$37 \quad 1.2(1.02,1.42)$

$38 \quad 1.21(1.02,1.43)$

$32 \quad 1.24(1.01,1.52)$

$8 \quad 1.04(1,1.08)$

$16 \quad 0.99(0.95,1.03)$

$16 \quad 0.99(0.95,1.02)$

$22 \quad 0.97(0.94,1)$

$22 \quad 0.97(0.95,1)$

$30 \quad 1.11(1,1.23)$

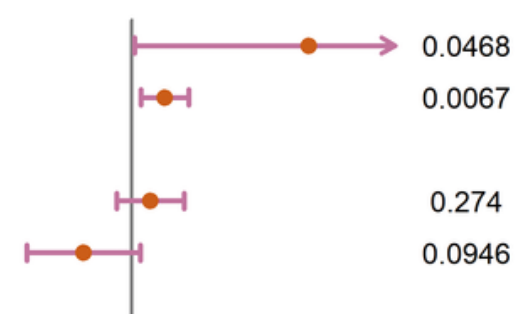

0.0946

0.0511

0.0017

0.0609

0.0694

0.0383

0.0154

0.022

0.0245

0.0234

0.0249

0.0258

0.026

0.0227

0.0262

0.0259

0.0374

0.0331

0.0317

0.0312

0.0431

0.0798

0.639

0.5012

0.0865

0.0307

0.0558

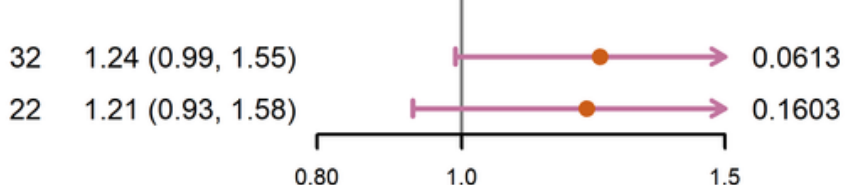

Effects of $1-S D$ increase in genetically determined metabolites on proxy-AD

\section{Figure 3}

Mendelian randomization results for associations between circulating metabolites and family history of Alzheimer's disease AD, Alzheimer's disease; $\mathrm{Cl}$, confidence interval; SD, standard deviation; SNPs, single nucleotide polymorphisms; OR, odds ratio. 


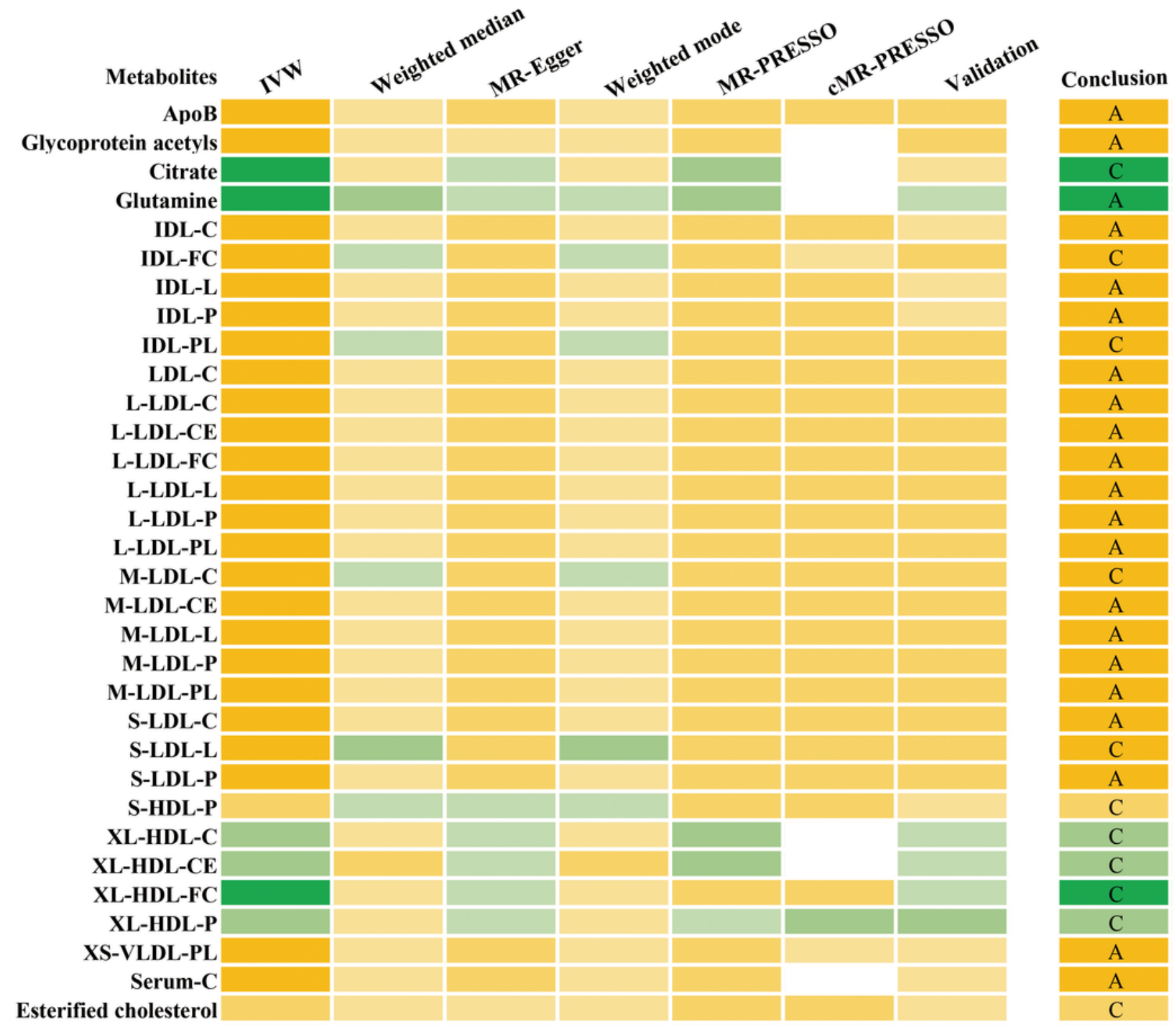

\begin{tabular}{|l|l|}
\hline & Protective effect on AD risk \\
\hline & Protective effect on AD risk with $\mathrm{P}<0.05$ \\
\hline & Protective effect on AD risk with $\mathrm{FDR} \mathrm{P}<0.05$ in IVW \\
\hline & Adverse effect on AD risk \\
\hline & Adverse effect on AD risk with $\mathrm{P}<0.05$ \\
\hline & Adverse effect on AD risk with FDR $\mathrm{P}<0.05$ in IVW \\
\hline $\boldsymbol{A}$ & Consistent evidence across main and sensitivity methods \\
\hline$B$ & Suggestive evidence, consistent across methods \\
\hline $\mathrm{C}$ & Conflicting evidence across methods \\
\hline
\end{tabular}

\section{Figure 4}

Overview results of this MR study on circulating metabolites and Alzheimer's disease. All results can be found in Fig. 2, Fig. 3, Table 1, and Additional file 1: Table S2. AD, Alzheimer's disease; cMR-PRESSO, corrected Mendelian randomization pleiotropy residual sum and outlier after removing of significant outliers; IVW, inverse variance-weighted; MR-PRESSO, Mendelian randomization pleiotropy residual sum and outlier; SD, standard deviation; SNPs, single nucleotide polymorphisms. 


\section{Supplementary Files}

This is a list of supplementary files associated with this preprint. Click to download.

- Additionalfile1.xlsx

- Additionalfile2.docx 